\title{
FIXED POINT THEOREMS IN PROBABILISTIC ANALYSIS
}

\author{
BY A. T. BHARUCHA-REID
}

1. Introduction. Probabilistic operator theory is that branch of probabilistic (or stochastic) analysis which is concerned with the study of operator-valued random variables (or, simply, random operators) and their properties. The development of a theory of random operators is of interest in its own right as a probabilistic generalization of (deterministic) operator theory; and just as operator theory is of fundamental importance in the study of operator equations, the development of probabilistic operator theory is required for the study of various classes of random equations.

Although several concrete examples of random operators and random operator equations have been around for a long time, the systematic study of probabilistic operator theory and its applications was initiated by the Prague school of probabilists under the direction of the late Antonín Špaček in the 1950's. They recognized that in using operator equations to model various systems (which is the heart of applied mathematics) it is usually not sufficient to consider only random initial data, it is also necessary to take into consideration the fact that the operators used to describe the behavior of systems may not be known exactly. For example, in the case of difference and differential operators the coefficients (constants or functions) might not be known exactly. One knows only their approximate values together with some measure of the possible error. In the case of integral operators, the kernel might not be known exactly; this being the case when either the integral equation is the primary model of a system, or when it is the equivalent formulation of a differential boundary value problem used as a model. In many studies workers use what might be termed mean coefficients or kernels, thereby casting their problems in the framework of deterministic operator equations. The main disadvantage of this approach is that, in general, a considerable amount of 'information' is lost concerning the behavior of the system. In the theory of random operator equations the coefficients or kernels are assumed from the outset to be random variables or random functions; and the solutions obtained (if they exist) are random functions whose dynamical and statistical properties can be studied.

It is of interest to remark that the distinction between a deterministic and probabilistic approach to the formulation of operator equations lies mainly in

This article is based on an invited address entitled, Probabilistic operator theory, presented at the 712th meeting of the American Mathematical Society, University of Florida, Gainesville, March 7, 1974; received by the editors February 2, 1976.

AMS (MOS) subject classifications (1970). Primary 60H99, 47H10.

Key words and phrases. Probabilistic analysis, fixed point theorems, random fixed point theorems, random operators, random differential and integral equations. 
the nature of the questions they try to answer, and in the interpretation of the results. The advantages of a probabilistic approach are that (1) it permits from the initial formulation a greater generality (and hence flexibility) than that offered by a deterministic approach, and (2) it permits the inclusion of probabilistic features in the equations, which may play an essential role in making the connection between operator equations and the real phenomena they purport to describe.

Research in probabilistic operator theory generally falls into one or more of the following areas: (1) operator-valued random variables and their properties, (2) operator-valued random functions (including semigroups of random operators) and their properties, (3) random equations whose solutions are operator-valued, (4) spectral theory of random operators, (5) measure-theoretic problems, (6) fixed point theorems, and (7) limit theorems. In my invited talk I was able to give a brief survey of research in all of the above areas; however in preparing this article I have elected to restrict my attention to just one of the areas-namely, fixed point theorems for random operators.

In $\$ 2$ we give some definitions of random operators, and give a few examples of random operators. We give only those definitions and concepts which are used in this article. For a detailed discussion of random operators and their properties we refer to [6]. $\S 3$ is devoted to the statement of three well-known fixed point theorems-namely, those of Banach, Schauder, and Krasnosel'ski1. In $\$ 4$, which is the main section of this article, we present an account of some random fixed point theorems which are probabilistic analogues of the theorems stated in $\S 3$. Finally, in $\$ 5$ we discuss briefly some current research and mention a few problems that might be investigated.

2. Random operators: Definitions and examples. Let $(\Omega, \mathcal{Q}, \mu)$ be a complete probability measure space; and let $(\mathcal{X}, \mathscr{B})$ and $(\mathcal{Y}, \mathcal{C})$ be two measurable spaces, where $\mathcal{X}$ and $\mathscr{Y}$ are Banach spaces of the same scalar type and $\mathscr{B}$ and $\mathcal{C}$ are the $\sigma$-algebras of Borel subsets of $\mathcal{X}$ and $\mathcal{Y}$, respectively. We first introduce the notion of a Banach space-valued random variable.

Definition 1. A mapping $x: \Omega \rightarrow \mathfrak{X}$ is said to be an $\mathfrak{X}$-valued random variable (random element, or generalized random variable) if the inverse image under the mapping $x$ of every $B \in \mathscr{B}$ belongs to $Q$; that is, $x^{-1}(B) \in \mathbb{Q}$ for all $B \in \mathscr{B}$.

Let $T$ be an operator from $\mathcal{X}$ to $\mathcal{Y}$ with domain $\mathscr{D}(T) \subset \mathcal{X}$. (We remark that, in general, $\mathscr{D}(T)$ will depend on $\omega$.

Definition 2. A mapping $T: \Omega \times \mathscr{D}(T) \rightarrow \mathcal{Y}$ is said to be a random operator if $y(\omega)=T(\omega) x$ is a $\mathcal{Y}$-valued random variable for every $x \in \mathcal{D}(T)$.

DEFINITION 3. A random operator $T(\omega)$ on $\mathcal{X}$ is said to be (a) linear if $T(\omega)\left[\alpha x_{1}+\beta x_{2}\right]=\alpha T(\omega) x_{1}+\beta T(\omega) x_{2}$ a.s. for all $x_{1}, x_{2} \in \mathcal{Q}(T), \alpha, \beta$ scalars; and (b) bounded if there exists a nonnegative real-valued random variable $M(\omega)$ such that for all $x \in \mathscr{D}(T),\|T(\omega) x\| \leqslant M(\omega)\|x\|$ a.s.

Definition 4. A random operator $T(\omega)$ on $\mathcal{X}$ is said to be (a) continuous at $x_{0}$ if $\lim _{n \rightarrow \infty}\left\|x_{n}-x_{0}\right\|=0$ implies $\lim _{n \rightarrow \infty}\left\|T(\omega) x_{n}-T(\omega) x_{0}\right\|=0$ a.s.; and 
(b) stochastically continuous if for every $x \in \mathscr{D}(T)$ and every $\varepsilon>0$, $\lim _{\|y-x\| \rightarrow 0} \mu(\{\omega:\|T(\omega) y-T(\omega) x\|>\varepsilon\}) \rightarrow 0$.

Let $\mathcal{L}(\mathcal{X})$ denote the Banach algebra of all endomorphisms of $\mathscr{X}$; and let $\Im$ denote the $\sigma$-algebra of Borel subsets of $\mathcal{L}(\mathcal{X})$, provided the norm topology is assumed.

Definition 5. A mapping $T: \Omega \rightarrow(\&, T)$ is said to be a random endomorphism of $\mathcal{X}$ if $T(\omega)$ is an $\mathcal{E}(\mathcal{X})$-valued random variable, that is $T^{-1}(F) \in \mathcal{Q}$ for every $F \in \mathcal{T}$.

We now introduce the notion of a separable random operator (cf. [44]), a notion that is useful in probabilistic operator theory (cf. Kannan and Salehi [32], Nashed and Salehi [46]).

Definition 6. A random operator $T(\omega)$ on a Banach space $\mathcal{X}$ is said to be separable if there exists a countable dense set $S$ in $\mathcal{X}$ and a negligible set $N \in \mathbb{Q}$ such that $\{\omega: T(\omega) x \in K, x \in F\} \triangle\{\omega: T(\omega) x \in K, x \in F \cap S\}$ $\subset N$ for every compact set $K$ and every open set $F$.

Let $H$ be a Hilbert space with inner product $(\cdot, \cdot)$. Random operators on $H$ to itself can, of course, be defined using Definitions 1 or 5; however the following definition is frequently employed.

Definition 7. A mapping $T: \Omega \times H \rightarrow H$ is said to be a random operator on $H$ if the function $\left(T(\omega) x_{1}, x_{2}\right)$ is a scalar-valued random variable for every $x_{1}, x_{2} \in H$.

We refer to Jajte [30] for an interesting measure-theoretic approach to the study of random operators on Hilbert spaces.

We now list a few examples of concrete random operators.

(i) RANDOM matrices. Let $\mathfrak{X}=R_{n}, n$ finite. An $n \times n$ random matrix $M(\omega)$ is a matrix whose elements $m_{i j}, i, j=1,2, \ldots, n$, are random variables; that is, $M(\omega)=\left(m_{i j}(\omega)\right)$. A random $n \times n$ matrix can also be defined as a mapping $M: \Omega \rightarrow \mathcal{L}\left(R_{n}\right)$, or $M: \Omega \rightarrow R_{n^{2}}$. Matrix-valued random functions arise in a number of applied problems. In these cases we have $M$ : $R^{+} \times \Omega \rightarrow \varrho\left(R_{n}\right)$. Random matrices constitute a very important class of random operators which are encountered in mathematical statistics (cf. Johnson and Kotz [31]) and physics (cf. Mehta [37]). Random matrices also arise in the study of systems of algebraic, difference, and differential equations with random coefficients.

(ii) RANDOM DIFFERENCE OPERATORS. Let $\mathfrak{X}=l_{2}$. A random difference operator on $l_{2}$ to itself can be defined as follows:

$$
T(\omega)\left[x_{k}\right]=\sum_{i=1}^{n} a_{i}(\omega) \tau^{i}\left[x_{k}\right]
$$

where $\tau^{i}$ denotes the translation operator $\tau^{i}\left[x_{k}\right]=x_{k+i}, i=0,1, \ldots, n$, and the $a_{i}(\omega)$ are real-valued random variables. Random difference operators arise in the study of discrete-parameter random dynamical systems; and many random difference equations have been studied in biology, economics, engineering, and time series analysis. 
(iii) RANDOM ORDINARY DifFERENTIAL OPERATORs. Let $\mathcal{X}=C[a, b]$, and let $C^{(n)}[a, b]$ denote the subspace of $C[a, b]$ consisting of all functions $x(t)$ whose first $n$ derivatives are continuous. We can define a random differential operator on $C^{(n)}$ to $C$ as follows:

$$
T(\omega)[x(t)]=\sum_{k=0}^{n} a_{k}(\omega) \frac{d^{k} x}{d t^{k}}, \quad t \in[a, b],
$$

where the coefficients $a_{k}(\omega)$ are real-valued random variables. In some applications the coefficients are real-valued random functions $a_{k}(t, \omega), t \in[a$, $b]$. Ordinary differential equations with random coefficients are perhaps the most widely studied class of random equations (cf. Soong [59]).

(iv) RANDOM PARTIAL DIFFERENTIAL OPERATORS. A number of concrete problems lead to the study, on appropriate function spaces, of random Schrödinger operators of the form

$$
S(\omega)[\psi]=\frac{\hbar^{2}}{2 m^{2}} \frac{\partial^{2} \psi}{\partial x^{2}}+V(\omega) \psi,
$$

where $V(\omega)$ is a random potential, and random diffusion operators of the form

$$
D(\omega)[u]=a(x, \omega) \frac{\partial^{2} u}{\partial x^{2}}+b(x, \omega) \frac{\partial u}{\partial x}+c(x, \omega) u .
$$

Random diffusion operators arise, for example, in the diffusion theory of gene frequencies (cf. Dale [15]). An interesting problem in probabilistic operator theory is to determine conditions which imply that random partial differential operators of the above form are infinitesimal generators of semigroups of random operators; that is, of operator-valued random functions $T$ : $R^{+} \times \Omega \rightarrow \varrho(X)$ such that $T\left(t_{1}, \omega\right) T\left(t_{2}, \omega\right)=T\left(t_{1}+t_{2}, \omega\right)$ a.s. and $T(0, \omega)=I$ a.s.

Random Helmholtz operators of the form $\Delta \psi+k_{0}^{2} n^{2}(r, \omega) \psi$, where $n^{2}$ is the index of refraction (assumed to be a random function), arise in the study of wave propagation in random media (cf. Frisch [17]).

(v) RANDOM INTEGRAL OPERATORS. Of the several classes of random equations which have been studied, random integral equations (and random differential equations formulated as random integral equations) have been studied most extensively (cf. Bharucha-Reid [6], and Tsokos and Padgett [62]). In particular, linear random Fredholm and Volterra operators of the form

$$
\begin{aligned}
& F(\omega)[f(x)]=\int_{a}^{b} K(x, y, \omega) f(y) d y \quad \text { and } \\
& V(\omega)[f(x)]=\int_{a}^{x} K(x, y, \omega) f(y) d y,
\end{aligned}
$$

as well as nonlinear random Uryson operators of the form

$$
U(\omega)[f(x)]=\int_{a}^{b} G(x, y, f(y), \omega) d y
$$

In these examples the limits of integration are given, but in many problems 
these limits themselves are random variables. We remark that an integral operator with a random kernel can often be expressed as an integral operator with a deterministic kernel, but the region over which the integral is defined is a random set.

3. Some well-known fixed point theorems. Let $\mathcal{X}$, be a Banach space, and let $T$ be an operator mapping $\mathcal{X}$ into itself.

Definition 8. An element $x \in \mathfrak{X}$ such that $T x=x$ is said to be a fixed point of $T$.

It is clear that the set of fixed points of an operator $T$ is the same as the solution set of the eigenproblem $(T-\lambda I) x=\theta$, corresponding to the eigenvalue $\lambda=1$. Consider a concrete operator equation $S x=y$ in a given Banach space. Then finding a fixed point of $T$ is equivalent to obtaining a solution of the operator equation $T x \equiv S x-x-y$.

In general, fixed point theorems fall into two classes: (1) topological fixed point theorems, and (2) algebraic, or constructive, fixed point theorems. Theorems of topological type are strictly existence theorems; that is, they establish conditions under which a fixed point exists but they do not provide a method for finding it. On the other hand, theorems of algebraic type give a method for finding a fixed point which can be called an iteration or successive approximation procedure.

In this section we state three well-known fixed point theorems which have been widely used in the theory of operator equations. For detailed discussions of these theorems we refer to Anselone [1], Bonsall [9], Rall [52], Saaty [53], and Smart [58]. For a bibliography of fixed point theorems and their applications we refer to Thompson [61, pp. 107-134]. Applications of these theorems in the theory of random equations will be indicated. Probabilistic analogues of these theorems will be given in the next section.

The prototype of most algebraic fixed point theorems is the contraction mapping theorem or principle due to Banach [2]. This is an abstract formulation of the classical method of successive approximation due to Picard. We recall that if $(\mathfrak{X}, d)$ is a complete metric space and $T$ is an operator with domain $\mathscr{D}(T)$ and range in $\mathcal{X}$, then $T$ is said to be a contraction operator if, for all $x, y \in \mathscr{D}(T), d(T x, T y) \leqslant k d(x, y)$, where $k \in[0,1)$.

THEOREM 1. If $T$ is a contraction operator mapping a complete metric space $(\mathfrak{X}, d)$ into itself, then $T$ has a unique fixed point (i.e., the equation $T x=x$ has one and only one solution). The fixed point, say, $\xi$, can be determined by the successive approximations $x_{n+1}=T x_{n}, n=0,1, \ldots$ (where $x_{0}$ is any arbitrary element of $\chi$ ) which converge to $\xi$. The error estimate is given by

$$
d\left(x_{n}, \xi\right) \leqslant\left(k^{n} /(1-k)\right) d\left(x_{0}, x_{1}\right) .
$$

Actually the contraction condition characterizes, in a certain sense, all mappings $\mathrm{T}$ from a complete metric space into itself such that for every $x$ in the space, the iterates $T^{n} x$ converge to the unique fixed point of $T$. The following result, due to Bessaga [3], clarifies this. 
THEOREM 2. Suppose $T$ is a mapping of an abstract set $\mathcal{X}$ into itself such that each iterate $T^{n}(n=1,2, \ldots)$ has a unique fixed point. Then for each $k \in(0$, 1) there exists a complete metric $d$ for $\mathcal{X}$ such that $T$ is a contraction mapping with contraction constant $k$.

A useful generalization of Theorem 1 is the following result (cf. Chu and Diaz [14], Kolmogorov and Fomin [35]):

THEOREM 3. If $T$ is an operator mapping a complete metric space $\mathcal{X}$ into itself, and if $T^{n}$ is a contraction for some $n$ (where $n$ is a positive integer), then $T$ has a unique fixed point.

Theorems 1 and 3 have found numerous applications in probabilistic analysis. In particular, Tsokos and Padgett [62] have used the Banach contraction mapping theorem to establish the existence and uniqueness of solutions of a number of random Fredholm and Volterra integral equations; and Theorem 3 has been used to establish existence and uniqueness of solutions of (i) classical Itô integral equations (cf. Bharucha-Reid [6]), (ii) Itô equations in Hilbert spaces (Cabaña [11]), and (iii) Itô equations in scales of Hilbert spaces (cf. [6]). Banach's theorem has also been used by Buche (cf. [6]) in his studies of nonlinear integral equations with random right-hand side.

As is well known, there are a large number of generalizations of Banach's theorem, and theorems of Banach type in the literature. To the best of my knowledge few if any of these have been used in probabilistic analysis.

The next theorem we consider is a topological fixed point theorem due to Schauder [54]. Schauder's theorem, which we now state, is the most important (for analysis) generalization of Brouwer's basic fixed point theorem.

THEOREM 4. Let $T$ be a continuous operator which maps a compact convex subset $E$ of a Banach space $\mathfrak{X}$ into $E$. Then there exists at least one element $\xi \in E$ such that $T \xi=\xi$.

The above theorem has been employed in probabilistic analysis by Tsokos and his colleagues (cf. [62]) who have used it to establish the existence of solutions of several classes of random integral equations.

Krasnosel'skiū's theorem has been used by Tsokos and Padgett [62] to establish the existence of solutions of random integral equations of mixed Volterra-Fredholm type.

Theorem 5. Let $\mathcal{X}$ be a Banach space, E a bounded, closed, convex subset of $\mathcal{X}$, and $S$ and $T$ operators on $E$ into $\mathcal{X}$ such that $S x_{1}+T x_{2} \in E$ for every pair $x_{1}, x_{2} \in E$. If $S$ is a contraction (i.e., $\left\|S x_{1}-S x_{2}\right\| \leqslant k\left\|x_{1}-x_{2}\right\|$ for all $x_{1}$, $x_{2} \in E$, for some $k \in[0,1)$, and $T$ is continuous and compact, then the operator equation $S x+T x=x$ has a solution $\xi \in E$.

Krasnosel'skiı̌'s theorem is of importance in proving existence theorems for perturbed operator equations. In particular, $S$ may be considered as perturbed by $T$, or $T$ might be considered as being perturbed by $S$. In either 
case, the theorems assert the existence of solutions of the perturbed operator equation.

Krasnosel'skiř's theorem has been used by Tsokos and Padgett [62] to establish the existence of solutions of random integral equations of mixed Volterra-Fredholm type.

In all of the above studies which have employed the Banach, Schauder, or Krasnosel'skiü's theorem the general procedure was the same; namely, to define an integral operator on an appropriate space of random functions, establish its general properties, and then show that the operator has the properties required for application of the thoerem.

4. Random fixed point theorems. Consider an operator equation of the form $T x=y$ in a concrete Banach space $\mathcal{X}$. Probabilistic analogues of such an equation are of three types: (1) $T x=y(\omega)$, in which the nonhomogeneous term, or forcing function, is random; (2) $T(\omega) x=y$, in which the operator is random and the nonhomogeneous term is known; and (3) $T(\omega) x=y(\omega)$, in which both the operator and the nonhomogeneous term are random. Equations of the first type are, in principle, the easiest to solve. Equations involving random operators are much more difficult to handle, and most of the research in probabilistic operator theory has been devoted to the development of techniques for solving random operator equations (cf. [6, Chapter 3]).

In this section we present an account of some of the random fixed point theorems that have been used to establish the existence, uniqueness, and measurability of solutions of random operator equations. Let $\mathcal{X}$ be a Banach space, and consider the equation $T(\omega) x(\omega)=y(\omega)$ in $\mathscr{X}$.

Definition 9. Any $x$-valued random variable $x(\omega)$ which satisfies the condition $\mu(\{\omega: T(\omega) x(\omega)=y(\omega)\})=1$ is said to be a random solution of the random operator equation $T(\omega) x(\omega)=y(\omega)$.

Definition 10. An $\chi$-valued random variable $\xi(\omega)$ is said to be a fixed point of the random operator $T(\omega)$ if $\xi(\omega)$ is a random solution of the equation $T(\omega) \xi(\omega)=\xi(\omega)$.

The study of fixed point theorems for random operators was initiated by Špaček [60] and Hanš [22]. The first systematic investigation of random fixed point theorems was carried out by Hanš [22]. In this section we state and prove several random fixed point theorems, and indicate some applications of these theorems.

Because of the wide applicability of Banach's contraction mapping theorem in the study of deterministic operator equations, Špaček and Hanš directed their attention to probabilistic versions of Banach's theorem and used their results to prove the existence, uniqueness, and measurability of solutions of Fredholm integral equations with random kernels.

We first introduce the notion of a random contraction operator.

Definition 11. A random operator $T(\omega)$ on a Banach space $\mathscr{X}$ with domain $\mathscr{D}(T(\omega))$ is said to be a random contraction operator if there exists a nonnegative real-valued random variable such that $k(\omega)<1$, and such that 
a.s. $\left\|T(\omega) x_{1}-T(\omega) x_{2}\right\| \leqslant k(\omega)\left\|x_{1}-x_{2}\right\|$ for all $x_{1}, x_{2} \in \mathscr{D}(T(\omega))$. If $k(\omega)$ $=k$ (a constant) for all $\omega \in \Omega$, then $T(\omega)$ is called a uniform random contraction operator.

The following theorem, due to Hanš [25], is a generalization of earlier results of Hanš and is a useful formulation for random contraction mapping theorems.

THEOREM 6. Let $\mathfrak{X}$ be a separable Banach space, and let $T(\omega)$ be a continuous random operator on $\mathcal{X}$ to itself such that

$$
\begin{array}{r}
\mu \bigcup_{m=1}^{\infty} \bigcup_{n=1}^{\infty} \bigcap_{x_{1} \in \mathcal{X}} \bigcap_{x_{2} \in \mathcal{X}}\left\{\omega:\left\|T^{n}(\omega) x_{1}-T^{n}(\omega) x_{2}\right\|\right. \\
\left.\left.\quad \leqslant\left(1-\frac{1}{m}\right)\left\|x_{1}-x_{2}\right\|\right\}\right)=1,
\end{array}
$$

where for every $\omega \in \Omega, x \in \mathcal{X}$, and $n=1,2, \ldots$, we put $T^{1}(\omega) x=T(\omega) x$, and $T^{n+1}(\omega) x=T(\omega)\left[T^{n}(\omega) x\right]$. Then, there exists an $\mathcal{X}$-valued random variable $\xi(\omega)$ which is the unique fixed point of $T(\omega)$; that is, if $\tilde{\xi}(\omega)$ is another fixed point, then $\xi(\omega)=\tilde{\xi}(\omega)$ a.s.

Proof. Let $E$ denote those elements of $\Omega$ belonging to the set

$$
\bigcup_{m=1}^{\infty} \bigcup_{n=1}^{\infty} \bigcap_{x_{1} \in \mathfrak{X}} \bigcap_{x_{2} \in \mathfrak{X}}\left\{\omega:\left\|T^{n}(\omega) x_{1}-T^{n}(\omega) x_{2}\right\| \leqslant\left(1-\frac{1}{m}\right)\left\|x_{1}-x_{2}\right\|\right\}
$$

for which $T(\omega)$ is continuous. Clearly $E \in \mathbb{Q}$, and, by hypothesis, $\mu(E)=1$. Let the mapping $\xi(\omega): \Omega \rightarrow \mathcal{X}$ be defined as follows: For every $\omega \in E, \xi(\omega)$ is equal to the unique fixed point of $T(\omega)$; and for every $\omega \in \Omega-E$, put $\xi(\omega)=\theta$ (the null element of $\mathfrak{X}$ ). Then $T(\omega) \xi(\omega)=\xi(\omega)$ a.s.

To establish the measurability of the fixed point $\xi(\omega)$ we proceed as follows. Let $x_{0}(\omega)$ be an arbitrary $\chi$-valued random variable, and put $x_{1}(\omega)=$ $T(\omega) x_{0}(\omega) . x_{1}(\omega)$ is an $\mathcal{X}$-valued random variable [6, Theorem 2.14], and a sequence of $x$-valued random variables can be defined as follows: $x_{n}(\omega)=$ $T(\omega) x_{n-1}(\omega), n=1,2, \ldots$ Now, since $T(\omega)$ is continuous, the sequence $x_{n}(\omega)$ converges almost surely to $\xi(\omega)$; hence [6, Theorem 1.6] $\xi(\omega)$ is an $\mathcal{X}$-valued random variable.

The uniqueness of the fixed point follows from the uniqueness of $\xi(\omega)$ for every $\omega \in E$.

We remark inat there is no difficulty in proving the measurability of the fixed point for random fixed point theorems of the constructive type, since the iterative process defines a sequence of $\mathfrak{X}$-valued random variables which converges a.s. to the fixed point. We will see that this is not the case when random fixed point theorems of a topological type are considered.

We now use Theorem 6 to prove the following random contraction mapping theorem. 
THEOREM 7. Let $T(\omega)$ be a continuous random operator on a separable Banach space $\mathcal{X}$ to itself, and let $k(\omega)$ be a nonnegative real-valued random variable such that $k(\omega)<1$ a.s. and $\left\|T(\omega) x_{1}-T(\omega) x_{2}\right\| \leqslant k(\omega)\left\|x_{1}-x_{2}\right\|$ for every pair of elements $x_{1}, x_{2} \in \mathcal{X}$. Then there exists an $\mathcal{X}$-valued random variable $\xi(\omega)$ which is the unique fixed point of $T(\omega)$.

Proof. Let $E=\{\omega: k(\omega)<1\}, F=\{\omega: T(\omega) x$ is continuous in $x\}$, and

$$
G_{x_{1}, x_{2}}=\left\{\omega:\left\|T(\omega) x_{1}-T(\omega) x_{2}\right\| \leqslant k(\omega)\left\|x_{1}-x_{2}\right\|\right\} .
$$

Since $\mathfrak{X}$ is separable, the intersections in the expression

$$
\bigcap_{x_{1} \in \mathfrak{X}} \bigcap_{x_{2} \in \mathfrak{X}}\left(G_{x_{1}, x_{2}} \cap E \cap F\right)
$$

can be replaced by intersections over a countable dense set of $\mathcal{X}$. Therefore the condition of Theorem 6 is satisfied with $n=1$.

Random contraction mapping theorems are of fundamental importance in the theory of random equations in that they can be used to establish the existence, uniqueness, and measurability of solutions of random operator equations. Probabilistic analogues of Banach's theorem have been used in the study of random linear and nonlinear integral equations (cf. Bharucha-Reid [4], [5], [6]). Random contraction mapping theorems can also be used to establish the existence of random solutions of the Fredholm integral equations obtained by Boyce [10] in his studies of differential equations with random boundary conditions. Random contraction mapping theorems have been utilized in the study of stochastic approximation procedures (cf. Gardner [19], Harıš [24], and Wasan [63]), and have been used by Oza [48] and Oza and Jury [49] to obtain an algorithm for the identification of a random linear discrete-time system described by a random difference equation. We refer to Grenander [20] and Sehgal [55] for other random contraction mapping theorems, and we refer to Driml and Hanš [16] and Hanš and Špaček [26] for some continuous analogues of random contraction mapping theorems and their applications.

We now state and prove an inversion theorem, due to Hanš [25], which utilizes Theorem 7. This theorem can be used to establish solutions of random eigenproblems of the form $(T(\omega)-\lambda I) x=y(\omega)$.

THEOREM 8. Let $T(\omega)$ be a random contraction operator on a separable Banach space $\mathcal{X}$, and let $k(\omega)$ be a nonnegative real-valued random variable which is bounded a.s. Then, for every real $\lambda \neq 0$ such that $k(\omega)<|\lambda|$ a.s. there exists a random operator $S(\omega)$ which is the inverse of $T(\omega)-\lambda I$.

Proof. Since $\lambda \neq 0, T(\omega)-\lambda I$ is invertible whenever the random operator $(1 / \lambda) T(\omega)-I$ is invertible, and vice versa. However, for every $y \in \mathcal{X}$ the random operator $T_{y}(\omega)$ defined, for every $\omega \in \Omega$ and $x \in \mathcal{X}$, by $T_{y}(\omega)[x]=$ $(1 / \lambda) T(\omega) x-y$ is a random contraction operator. Therefore, by Theorem 7 , there exists a unique random fixed point $\xi_{y}(\omega)$ satisfying the relation $\xi_{y}(\omega)=$ $(1 / \lambda) T(\omega) \xi_{y}(\omega)-y$ a.s. However, the above statement is equivalent to the 
invertibility of the random operator $(1 / \lambda) T(\omega)-I$, and therefore the invertibility of the random operator $T(\omega)-\lambda I$.

The next random fixed point theorem we consider, which is due to Mukherjea [40], [41], is a probabilistic version of Schauder's theorem.

THEOREM 9. Let $(\Omega, Q, \mu)$ be an atomic probability measure space, and let $E$ be a compact (or closed and bounded) convex subset of a separable Banach space $\mathcal{X}$. Let $T(\omega)$ be a compact random operator mapping $E$ into itself. Then, there exists an $E$-valued random variable $\xi(\omega)$ such that $T(\omega) \xi(\omega)=\xi(\omega)$ a.s. $n$, such that $T\left(\omega_{n}\right) \varphi_{n}=\varphi_{n}$. Put $\xi(\omega)=\varphi_{n}$ for $\omega \in C_{n}$, and 0 otherwise. Then $T(\omega) \xi(\omega)=\xi(\omega)$.

A more interesting theorem is the following, due to Bharucha-Reid and Mukherjea.

THEOREM 10. Let E be a compact convex subset of a Banach space and $T(\omega)$ be a continuous random operator mapping $E$ into itself. Then there exists an E-valued random variable $\xi(\omega)$ such that $T(\omega) \xi(\omega)=\xi(\omega)$ a.s.

Proof. Let $A(\omega)=\{x \in E: T(\omega) x=x\}$. Then by Theorem 4, for each $\omega$ the set $A(\omega)$ is nonempty. Furthermore, for any closed subset $F$ of $E$

$$
\begin{aligned}
\{\omega: A(\omega) \cap F \text { is nonempty }\} & =\{\omega: A(\omega) x=x \text { for some } x \text { in } F\} \\
& =\bigcap_{n=1}^{\infty} \bigcup_{i=1}^{\infty}\left\{\omega:\left\|T(\omega) x_{i}-x_{i}\right\|<1 / n\right\}
\end{aligned}
$$

where the $x_{i}$ 's form a dense sequence in $F$. It is therefore clear that the set $\{\omega$ : $A(\omega) \cap F$ is nonempty $\}$ is measurable for every closed subset $F$ of $E$. To prove the theorem, it is sufficient to find an $E$-valued random variable $\xi(\omega)$ such that $\xi(\omega) \in A(\omega)$. It is known that we can associate with the space $E$ a sequence of triples $\left(C_{n}, P_{n}, \Phi_{n}\right)(n$ a nonnegative integer) such that

(i) Each $C_{n}$ is a countable set and $P_{n}$ maps $C_{n+1}$ onto $C_{n}$;

(ii) $\Phi_{n}$ maps $C_{n}$ into a class of nonempty closed subsets of $E$ of diameter $\leqslant$ $2^{-n}$;

(iii) $E=\cup_{c \in C_{0}} \Phi_{0}(c)$;

(iv) for each $n$ and for each $c$ in $C_{n}$,

$$
\Phi_{n}(c)=\bigcup_{P_{n} c^{\prime}=c} \Phi_{n+1}\left(c^{\prime}\right) .
$$

We assume with no loss of generality that $C_{0}$ and each $P_{n}^{-1}(c)$ with $c$ in $C_{n}$ are natrurally linearly ordered such that only finitely many elements can precede any element in this order. We now use an idea of Castaing [13]. For each $n$, we intend to find a suitable partition of $\Omega$. We proceed inductively as follows: For each $c$ in $C_{0}$, we define $\Omega_{c}$ by $\omega \in \Omega_{c}$ if and only if $A(\omega) \cap \Phi_{0}(c)$ is nonempty and $A(\omega) \cap \Phi_{0}\left(c^{\prime}\right)$ is empty for $c^{\prime} \in C_{0}, c^{\prime}<c$. Then the $\Omega_{c}$ 's are pairwise disjoint measurable sets with union $\Omega$. Suppose now that we have found a partition of $\Omega$ corresponding to the elements of $C_{k}$. To do this for 
$C_{k+1}$, we define for $c$ in $C_{k+1}$ the set $\Omega_{c}$ by $\omega \in \Omega_{c}$ if and only $\omega \in \Omega_{P_{k}(c)}$ and $A(\omega) \cap \Phi_{k+1}(c)$ is nonempty, but $A(\omega) \cap \Phi_{k+1}\left(c^{\prime}\right)$ is empty for $c^{\prime}$ in $P_{k}^{-1}\left(P_{k}(c)\right)$ and $c^{\prime}<c$.

For any positive $n$ and each $c \in C_{n}$ we choose an element $x_{n}(c) \in \Phi_{n}(c)$ and define $\xi_{n}(\omega)=x_{n}(c)$ for $\omega \in \Omega_{c}$ where the $\Omega_{c}$ 's are members of the partition of $\Omega$ corresponding to the elements of $C_{n}$. Then each $\xi_{n}(\omega)$ is measurable and

$$
\left\|\xi_{n}(\omega)-\xi_{n+1}(\omega)\right\| \leqslant 2^{-n}, \quad d\left(\xi_{n}(\omega), A(\omega)\right) \leqslant 2^{-n} .
$$

Therefore if $\xi(\omega)=\lim \xi_{n}(\omega)$, then $\xi(\omega) \in A(\omega)$ and the theorem follows. (Note that in this theorem $E$ could be assumed to be a compact subset of a metric space instead of a Banach space if each $A(\omega)$ was guaranteed to be nonempty by some other conditions.)

Theorem 9 has been used by Mukherjea [43] (cf. also [6]) to prove the existence of a solution of a random Uryson integral equation in $C[0,1]$ of the form

$$
\int_{I(\omega)} K(t, \tau, x(\tau, \omega), \omega) d \tau-x(t, \omega)=y(t, \omega),
$$

where $I(\omega)=[a(\omega), b(\omega)] \subset[0,1]$; that is, $I(\omega)$ is a subset of the interval $[0$, 1], where $a(\omega)$ and $b(\omega)$ are real-valued random variables. Mittermeier [38] has used Mukherjea's theorem to establish the existence of a random equilibrium price vector in his analysis of a random exchange economy.

It is clear that in probabilistic analogues of classical fixed point theorems of topological type, the main difficulty is in establishing the measurability of a fixed point. Thus to avoid certain difficulties in Theorem 10, $E$ was assumed to be compact (not just closed and bounded) and the random operator was assumed continuous. However, with little extra work some of these conditions can be relaxed as Theorem 11 will indicate.

We now state another probabilistic version of Schauder's theorem due to Kannan and Salehi [32].

THEOREM 11. Let $T(\omega)$ be a stochastically continuous random operator on a separable Banach space $\mathfrak{X}$ to itself. Suppose that for each $\omega \in \Omega,\{x: T(\omega) x=$ $x\} \neq \varnothing$ (the null set). Then there exists a measurable multivalued map $\xi(\omega)$ : $\Omega \rightarrow 2^{\text {X }}$ such that $\xi(\omega)=\{x: T(\omega) x=x\}$ a.s.

In a number of applications of fixed point theorems in probabilistic analysis, it is assumed that a random operator $T(\omega)$ satisfies the hypotheses of Schauder's theorem for each $\omega \in \Omega$. Then, if $T(\omega)$ is a continuous random operator it is also stochastically continuous and separable; hence the Kannan-Salehi theorem corresponds to a probabilistic analogue of Schauder's theorem. Theorem 11 can be utilized to establish the existence of solutions of random nonlinear differential and integral equations with monotonic nonlinearities (cf. [33], [34]).

The next random fixed point theorem we consider is a probabilistic version of Krasnosel'skiir's theorem originally due to Prakasa Rao [51] who initially 
assumed the probability space to be atomic.

THEOREM 12. Let $(\Omega, Q, \mu)$ be a probability measure space, and let $E$ be a compact and convex subset of a separable Banach space $\mathcal{X}$. Let $T(\omega)$ and $S(\omega)$ be continuous random operators mapping $\mathcal{X}$ into itself such that (i) $S(\omega) x_{1}+$ $T(\omega) x_{2} \in E$ for all $x_{1}, x_{2} \in E$ and $\omega \in \Omega$, (ii) there exists a nonnegative real-valued random variable $k(\omega)$ such that $\left\|S(\omega) x_{1}-S(\omega) x_{2}\right\| \leqslant k(\omega) \| x_{1}-$ $x_{2} \|$ for all $x_{1}, x_{2} \in E$ and $k(\omega)<1$ a.s. Then there exists an $\mathcal{X}$-valued random variable $\xi(\omega)$ such that $S(\omega) \xi(\omega)+T(\omega) \xi(\omega)=\xi(\omega)$ for all $\omega \in \Omega$.

The proof of the above theorem follows easily from Theorem 10, observing that the operator $[I-S(\omega)]^{-1} T(\omega)$ is a well-defined continuous mapping on $E$ into itself. Theorem 12 has been used by Prakasa Rao [51] to establish the existence of a solution of a random integral equation of the form

$$
\begin{aligned}
x(t, \omega)= & \int_{0}^{t} K_{1}(t, \tau, x(\tau, \omega), \omega) d \tau \\
& +\int_{0}^{T} K_{2}(t, \tau, x(\tau, \omega), \omega) d s+y(t, \omega), \quad t \in[0, T] .
\end{aligned}
$$

The last random fixed point theorem we consider, due to Mukherjea [42], is based on the concept of a resolvent, a notion analogous to the classical concept of the resolvent of a kernel in the theory of integral equations.

THEOREM 13. Let $T(\omega)$ be a random continuous linear operator on a separable Banach space $\mathfrak{X}$, and let $y(\omega)$ be an $X$-valued random variable. Then the random equation $T(\omega) x(\omega)+y(\omega)=x(\omega)$ has, for every $y(\omega)$, a unique random solution $x(\omega)$ for every $\omega \in \Omega$ if and only if there exists a random linear operator $S(\omega)$ such that for every $\xi \in \mathfrak{X}, T(\omega) \xi+S(\omega) \xi=T(\omega)[S(\omega) \xi]$, and $S(\omega)-I$ is onto for each $\omega \in \Omega$. Moreover, in the 'if' part of the above statement the measurability of $S(\omega)$ is not needed, and it is sufficient for the above conditions to hold for almost all $\omega$.

An immediate consequence of Theorem 13 is the following result in the theory of random integral equations (cf. [42]).

THEOREM 14. Suppose that for almost all $\omega \in \Omega$ the kernel $K(t, \tau, \omega)$ is in $C([a, b] \times[a, b])$, and for each $t, \tau \in[a, b]$ the kernel is a random variable. Assume also that for almost all $\omega \in \Omega, K(t, \tau, \omega)$ has a resolvent $R(t, \tau, \omega)$, which means that $R(\cdot, \tau, \omega)$ and $R(t, \cdot, \omega)$ are in $L_{2}[a, b]$ for all $\tau$ and $t$, respectively, and, moreover, $\int_{a}^{b} \int_{a}^{b}|R(t, \tau, \omega)|^{2} d t d \tau<\infty$ and

$$
\begin{aligned}
K(t, \tau, \omega)+R(t, \tau, \omega) & =\int_{a}^{b} K(t, \xi, \omega) R(\xi, \tau, \omega) d \xi \\
& =\int_{a}^{b} R(t, \xi, \omega) K(\xi, \tau, \omega) d \xi
\end{aligned}
$$

Then $R$ is a $C([a, b] \times[a, b])$-valued random variable, and the random Fredholm equation 


$$
x(t, \omega)=y(t, \omega)+\int_{a}^{b} K(t, \tau, \omega) x(\tau, \omega) d \tau
$$

has a unique random solution

$$
x(t, \omega)=y(t, \omega)-\int_{a}^{b} R(t, \xi, \omega) y(\xi \omega) d \xi
$$

for every $y(t, \omega)$ with values in $C([a, b])$.

5. Current research and some possible research problems. In this section we discuss briefly some current research on random fixed point theorems and mention a few problem areas that might be investigated.

a. It would be of interest to obtain probabilistic analogues of (i) fixed point theorems for nonexpansive $\left(d\left(T x_{1}, T x_{2}\right) \leqslant d\left(x_{1}, x_{2}\right)\right)$ and contractive or shrinking $\left(d\left(T x_{1}, T x_{2}\right)<d\left(x_{1}, x_{2}\right)\right)$ mappings, (ii) the Bohnenblust-Karlin Banach space generalization of Kakutani's fixed point theorem (cf. [8]), ${ }^{1}$ (iii) the theorems of Nashed and Wong of Krasnosel'skii type for nonlinear contractions (cf. [47]), ${ }^{2}$ and (iv) some of the many results presented by Petryshyn [50]. Useful probabilistic analogues of the above should find widespread applications in probabilisitc analysis.

b. Let $T(\omega)$ be a random contraction operator on a Banach space $\mathcal{X}$, and let $\xi(\omega)$ be its unique fixed point. $\xi(\omega)$ is an $\mathcal{X}$-valued random variable; hence if its Bochner integral exists we can define the expectation of the random fixed point, that is

$$
\bar{\xi}=\mathcal{E}\{\xi(\omega)\}=(\mathrm{B}) \int_{\Omega} \xi(\omega) d \mu .
$$

Similarly, if $T(\omega)$ is a uniform random contraction operator on $\mathcal{X}$, and if $T(\omega) x$ is Bochner integrable, then we can define the expectation of $T(\omega)$; that is

$$
S x=\mathscr{E}\{T(\omega) x\}=(\mathrm{B}) \int_{\Omega} T(\omega) x d \mu .
$$

Clearly $S$ is a contraction operator on $\mathcal{X}$; hence it has a unique fixed point, say $\psi$-which we call the fixed point of the expectation of $T(\omega)$. A question of great interest is the following: Under what conditions on $T$ is $\bar{\xi}=\psi$ ? Consider the random operator $T(\omega)$ defined for every $\omega \in \Omega$ and $x \in \mathcal{L}$ as follows: $T(\omega)[x]=\alpha x+z(\omega)$, where $\alpha<1$ and $z(\omega)$ is an $\mathcal{X}$-valued random variable whose expectation exists. In this case it is clear that $\bar{\xi}=\psi$; however, in general the expectation of a random fixed point does not coincide with the fixed point of the deterministic operator which arises as the expectation of the given random operator. We refer to Morse [39] for a discussion of this problem, and for some numerical results for a nonlinear random integral equation.

It is of interest to determine conditions such that equality obtains, as this would shed light on the problem of when the expectation of the solution of a

\footnotetext{
${ }^{1}$ R. Kannan has recently given a probability analogue of Kakutani's theorem.
}

${ }^{2}$ The probabilistic analogue in this case can now be obtained from Theorem 12. 
random operator equation is equal to the solution of the deterministic operator equation which results from averaging.

c. An area of active research is concerned with the study of fixed point theorems for mappings on probabilistic metric spaces. A probabilistic metric space $(P M$-space $)$ is an ordered pair $(\mathcal{X}, F)$, where $\mathcal{X}$ is an abstract set of elements and $F$ is a mapping of $\mathcal{X} \times \mathcal{X}$ into the space of all probability distribution functions. For $p, q \in \mathcal{X}$ we denote the distribution function $F(p, q)$ by $F_{p, q}$, and $F_{p, q}(\xi)$ represents the value of $F_{p, q}$ at $\xi \in R$. The functions $F_{p, q}$ are assumed to satisfy the following conditions:

(1) $F_{p, q}(\xi)=1$ for all $\xi>0$, if and only if $p=q$,

(2) $F_{p, q}(0)=0$,

(3) $F_{p, q}=F_{q, p}$,

(4) if $F_{p, q}\left(\xi_{1}\right)=1$ and $F_{q, r}\left(\xi_{2}\right)=1$, then $F_{p, r}\left(\xi_{1}+\xi_{2}\right)=1$.

The study of contraction mapping theorems for $P M$-spaces was initiated by Sehgal [55] (cf. also [56], [57]) who obtained analogues of the Banach contraction mapping theorem and other fixed point theorems for contraction mappings. More recently, Bocşan [7], Cain and Kasriel [12], Istrăţescu and Rovenţa [28], and Istrăţescu and Săcuiu [29], as well as other members of the research group at the University of Timişoara in Roumania have proved a number of interesting fixed point theorems for mapping on various classes of $P M$-spaces. Some of these results should be useful in studying the existence of solutions of operator equations in $P M$-spaces.

d. In the study of approximate solutions of random operator equations it would be of use to have theorems of Bonsall-Nadler-type (cf. [9], [45]) for random contraction operators. That is, one considers the following question: In a Banach space does the convergence (in some sense) of a sequence $\left\{T_{n}(\omega)\right\}$ of random contraction operators to a random contraction operator $U(\omega)$ imply the convergence (in some sense) of the associated sequence $\left\{\xi_{n}(\omega)\right\}$ of random fixed points to the random fixed point, say $\varphi(\omega)$, of $U(\omega)$ ?

In general, research needs to be done utilizing the additive and multiplicative limit theorems for random operators (cf. [6], [18], [20], [21], [23], [24]) in the study of limit theorems for random operators and the limiting behavior of their fixed points. Some results along these lines have been obtained by Hanš [24] using strong laws of large numbers for $\mathcal{X}$-valued random variables.

\section{REFERENCES}

1. P. M. Anselone (Ed.), Nonlinear integral equations, Univ. of Wisconsin Press, Madison, Wisconsin, 1964.

2. S. Banach, Sur les opérations dans les ensembles abstraits et leur applications aux équations intégrales, Fund. Math. 3 (1922), 133-181.

3. C. Bessaga, On the converse of the Banach "fixed-point principle", Colloq. Math. 7 (1959), 41-43. MR 22 \# 1882.

4. A. T. Bharucha-Reid, On random solutions of integral equations in Banach spaces, Trans. 2nd. Prague Conf. on Information Theory, Statist. Decision Functions and Random Processes (1959), Czechoslovak Acad. Sci., Prague, 1960; Academic Press, New York, 1961, pp. 27-48. MR 26 \#2836. 
5. _ On the theory of random equations, Proc. Sympos. Appl. Math., vol. 16, Amer. Math. Soc., Providence, R. I., 1964, pp. 40-69. MR 32 \#6498.

6. __ Random integral equations, Academic Press, New York, 1972.

7. G. Bocsan, On some fixed point theorems in probabilistic metric spaces, Seminar on the Theory of Functions and Applied Mathematics, A: Probabilistic Metric Spaces, No. 24, Univ. of Timişoara, 1974.

8. H. F. Bohnenblust and S. Karlin, On a theorem of Ville, Contribution to the Theory of Games, Vol. I, (H. W. Kuhn and A. W. Tucker, Eds.), Princeton Univ. Press, Princeton, N. J., 1950, pp. 155-160. MR 12, 844.

9. F. F. Bonsall, Lectures on some fixed point theorems of functional analysis, Tata Institute of Fundamental Research, Bombay, 1962. MR 33 \#6332.

10. W. E. Boyce, Random eigenvalue problems, Probabilistic Methods in Applied Mathematics (A. T. Bharucha-Reid, Ed.), Academic Press, New York, 1968, Vol. 1, pp. 1-73. MR 41 \#776.

11. E. M. Cabaña, Stochastic integration in separable Hilbert spaces, Univ. Repúb. Fac. Ingen. Agrimens. Montevideo Publ. Inst. Mat. Estadist. 4 (1966), 49-80. MR 33 \# 5001.

12. G. L. Cain and R. H. Kasriel, Fixed and period points of local contraction mappings on probabilistic metric spaces, Math. Systems Theory 9 (1976), 289-297.

13. C. H. Castaing, Sur les multi-applications mesurables, Rev. Française Informat. Recherche Opérationnelle 1 (1967), no. 1, 91-126. MR 36 \#6575.

14. S. C. Chu and J. B. Diaz, Remarks on a generalization of Banach's principle of contraction mappings, J. Math. Anal. Appl 11 (1965), 440-446. MR 32 \# 1691.

15. A. I. Dale, Some theoretical aspects of random equations of evolution in theoretical population genetics, Ph. D. Dissertation, Virginia Polytechnic Inst. and State Univ., Blacksburg, Virginia, 1972.

16. M. Driml and O. Hanš, Continuous stochastic approximations, Trans. 2nd. Prague Conf. on Information Theory, Statist. Decision Functions, and Random Processes (1959), Czechoslovak Acad. Sci., Prague, 1960; Academic Press, New York, 1961, pp. 113-122. MR 24 \# A562.

17. U. Frisch, Wave propagation in random media, Probabilistic Methods in Applied Mathematics (A. T. Bharucha-Reid, Ed.), Academic Press, New York, 1968, Vol. 1, pp. 75-198. MR 42 \#4088.

18. H. Furstenberg and H. Kesten, Products of random matrices, Ann. Math. Statist. 31 (1960), 457-469. MR 22 \# 12558.

19. L. A. Gardner, Jr., Stochastic approximation and its application to problems of prediction and control synthesis, Internat. Sympos. Nonlinear Differential Equations and Nonlinear Mechanics (J. P. LaSalle and S. Lefschetz, Eds.), Academic Press, New York, 1963, pp. 241-258. MR 26 \#5708.

20. U. Grenander, Probabilities on algebraic structures, Wiley, New York; Almqvist and Wiksell, Stockholm, 1963. MR 34 \#6810.

21. J. Hainis, Random variables with values in Banach algebras and random transformations in Hilbert spaces, Bull. Soc. Math. Grèce (N.S.) 7 (1966), fasc. 2, 179-223. (Greek. French summary) MR 37 \#3609.

22. O. Hanš, Reduzierende zufällige Transformationen, Czechoslovak Math. J. 7 (82) (1957), 154-158. MR 19, 777.

23. _ Generalized random variables, Trans. 1st Prague Conf. on Information Theory, Statist. Decision Functions, and Random Processes (Liblice, 1956), Czechoslovak Acad. Sci., Prague, 1957, pp. 61-103. MR 20 \#331.

24. - Random fixed point theorems, Trans. 1st Prague Conf. on Information Theory, Statist. Decision Functions, and Random Processes, (Liblice, 1956), Czechoslovak Acad. Sci., Prague, 1957, pp. 105-125. MR 20 \#7332.

$25 . \quad$, Random operator equations, Proc. 4th Berkeley Sympos. on Math. Statist. and Probability (1960), Vol. II, Univ. California Press, Berkeley, Calif., 1961, pp. 185-202. MR 26 \#4185.

26. O. Hanš and A. Špaček, Random fixed point approximation by differentiable trajectories, Trans. 2nd. Prague Conf. Information Theory, Statist. Decision Functions, and Random Processes (1959), Czechoslovak Acad. Sci., Prague, 1960; Academic Press, New York, 1961, pp. 203-213. MR 23 \# A1401.

27. V. Istrăţescu, An introduction to probabilistic metric spaces with applications, Editura Tehnică, Bucharest, 1974. (Roumanian) 
28. I. Istrăţescu and E. Rovenţa, On fixed point theorems for mappings on probabilistic metric spaces, Seminar on the Theory of Functions and Appl. Math., A: Probabilistic Metric Spaces, No. 19, Univ. of Timisoara, 1974.

29. V. I. Istrăţescu and I. Săcuiǔ, Fixed point theorems for contraction mappings on probabilistic metric spaces, Rev. Roumaine Math. Pures Appl. 18 (1973), 1375-1380. MR 48 \# 9690.

30. R. Jajte, Random linear operators, Bull. Acad. Polon. Sci. Sér. Sci. Math. Astronom. Phys. 21 (1973), 227-231. MR 47 \#9344.

31. N. L. Johnson and S. Kotz, Distributions in statistics: Continuous multivariate distributions, Wiley, New York, 1972.

32. R. Kannan and $\mathrm{H}$. Salehi, Mesurabilité du point fixe d'une transformation aléatoire séparable, C. R. Acad. Sci. Paris Sér A-B 281 (1975), A663-A664.

33. _ Random nonlinear equations and monotonic nonlinearities, J. Math. Anal. Appl. (to appear).

34. Measurability of solutions of nonlinear equations (to appear).

35. A. N. Kolmogorov and S. V. Fomin, Introductory real analysis, rev. ed; English transl. of Elements of the theory of functions and of functional analysis, Prentice-Hall, Englewood Cliffs, $\mathrm{N}$. J., 1970. MR 42 \# 1954.

36. M. A. Krasnosel'skiī, Two remarks on the method of successive approximations, Uspehi Mat. Nauk 10 (1955), no. 1 (63), 123-127. (Russian) MR 16, 833.

37. M. L. Mehta, Random matrices and the statistical theory of energy levels, Academic Press, New York and London, 1967. MR 36 \#354.

38. H. Mittermeier, $A$ note on random preference and equilibrium analysis: Application of $a$ stochastic fixed point theorem, Unpublished manuscript, 1974.

39. W. L. Morse, Some mathematics in stream temperature modelling (to appear).

40. A. Mukherjea, Random transformations on Banach spaces, Ph. D. Dissertation, Wayne State Univ., Michigan, 1966.

41. , Transformations aléatoires séparables: Théorème du point fixe aléatoire, C. R. Acad. Sci. Paris Sér A-B 263 (1966), A393-A395. MR 35 \#2336.

42. , On the measurability of the resolvent of a random kernel, Bull. Math. Soc. Sci.

Math. R. S. Roumanie (N. S.) 14 (62) (1970), 55-59 (1971). MR 48 \#7376.

43. On a random integral equation of Uryson type. I (to appear).

44. A. Mukherjea and A. T. Bharucha-Reid, Separable random operators. I, Rev. Roumaine Math. Pures Appl. 14 (1969), 1553-1561. MR 41 \#2732; erratum, 42, p. 1824.

45. S. B. Nadler, Jr., Sequences of contractions and fixed points, Pacific J. Math. 27 (1968), 579-585. MR 39 \#2147.

46. M. Z. Nashed and H. Salehi, Measurability of generalized inverses of random linear operators, SIAM J. Appl. Math. 25 (1973), 681-692. MR 48 \#12124.

47. M. Z. Nashed and J. S. W. Wong, Some variants of a fixed point theorem of Krasnosel'skil and applications to nonlinear integral equations, J. Math. Mech. 18 (1969), 767-777. MR 38 \#6416.

48. K. G. Oza, Identification problems and random contraction mappings, Ph. D. Dissertation, Univ. of California, Berkeley, 1967.

49. K. G. Oza and E. I. Jury, System identification and the principle of random contraction mapping, SIAM J. Control 6 (1968), 244-257. MR 38 \#5486.

50. W. V. Petryshyn, On the approximation-solvability of equations involving A-proper and pseudo-A-proper mappings, Bull. Amer. Math. Soc. 81 (1975), 223-312.

51. B. L. S. Prakasa Rao, Stochastic integral equations of mixed type. II, J. Mathematical and Physical Sci. 7 (1973), 245-260. MR 50 \# 14933.

52. L. B. Rall, Computational solution of nonlinear operator equations, Wiley, New York, 1969. MR 39 \#2289.

53. T. L. Saaty, Modern nonlinear equations, McGraw-Hill, New York, 1967. MR 36 \# 1249.

54. J. Schauder, Der Fixpunktsatz in Funktionenräumen, Studia Math. 2 (1930), 171-182.

55. V. M. Sehgal, Some fixed point theorems in functional analysis and probability, $\mathrm{Ph}$. D. Dissertation, Wayne State Univ., Michigan, 1966.

56. V. M. Sehgal and A. T. Bharucha-Reid, Fixed points of contraction mappings on probabilistic metric spaces, Math. Systems Theory 6 (1972), 97-102. MR 46 \#9956.

57. H. Sherwood, Complete probabilistic metric spaces, Z. Wahrscheinlichkeitstheorie und Verw. Gebiete 20 (1971), 117-128. MR 47 \#5837. 
58. D. R. Smart, Fixed point theorems, Cambridge Univ. Press, Cambridge, 1974.

59. T. T. Soong, Random differential equations in science and engineering, Academic Press, New York, 1973.

60. A. Spaček, Zufällige Gleichungen, Czechoslovak Math. J. 5 (80) (1955), 462-466. MR 18, 157.

61. R. B. Thompson (Ed.), Collection of articles on fixed point theory, (The Rocky Mountain Mathematics Consortium, Arizona State Univ., Tempe, 1974), Rocky Mountain J. Math. 4 (1974), no. 1, 1-29. MR 48 \#976.

62. C. P. Tsokos and W. J. Padgett, Random integral equations with applications to life sciences and engineering, Academic Press, New York, 1974. MR 50 \# 14934.

63. M. T. Wasan, Stochastic approximation, Cambridge Univ. Press, London and New York, 1969. MR $40 \# 975$.

Department of Mathematics, Wayne State University, Detroit, Michigan 48202 (Current address) 